\title{
Forrester's Concept in Modeling Heart Dynamics
}

\author{
Shmid* A.V. D.SC, Novopashin* M.A., Berezin* A.A. PhD \\ EC-leasing Moscow. Berezin A.A.
}

\begin{abstract}
The paper deals with the Forrester's approach to analysis of heart electrical dynamics based on the hypothesis that heart belongs to the class of Complex Systems and its dynamics can be described by coupled Van der Pol differential equations with a time lag. The chain of such equations suggested by Ginzburg and Landau was used to describe such states of heart dynamics as normal beatings, fibrillation and ischemia through the kinds of the chain dynamics as regular oscillations, chaotic dynamics and intermittency dynamics of order - disorder type correspondingly. The mathematical model supporting the hypothesis demonstrated all mentioned model modes of heart functioning. During the computer, study of the model another hypothesis has been put forward concerning the existence in the heart dynamics a special oscillatory region, which interacts with ischemia oscillatory region lowering its energy. According to the prediction, both regions had to have different resonant parameters. Taking into account the results of the model study there has been made another prediction about possible synchronization of the heartbeats by applying external periodical perturbation at frequency of about $1 \mathrm{~Hz}$. The pilot data supporting the proposed hypothesis have demonstrated the reality of the suggested hypothesis. In particular, the real ECG and their Fourier spectra of healthy patients corresponded to the model regular ECG and their Fourier spectra. The ECG Fourier spectrum of the patient with a cardio stimulator corresponded to the model synchronization of the ECG and its Fourier spectrum generated by the developed mathematical model. As far as the ECGs and Fourier spectra in patients with differently developed ischemia are concerned, they confirmed the hypothesis about the existing of two interacting oscillatory regions in myocardium: the ischemic and the defending ones. The defending region decreases the volume of the ischemic process through lowering its energy. Summing it up one can conclude that the experimental results confirmed the suggested hypothesis and the prediction following from it.
\end{abstract}

\section{Introduction}

All biological systems are known to be thermodynamically open and having many degrees of freedom. According to J. Forrester [1] such systems are called Complex Systems. All Complex Systems can be characterized by ambiguous reactions to external influence, and this is the main reason for failures of the attempts undertaken from the outside with the purpose to improve the behavior of the system. The term « Complex System » means in itself iterative structure of the big order with a nonlinear feedback. Complex System has a number of specific features. Any Complex System is a system of a big order. "Order" of the System is defined by the number of differential equations necessary for the description of its levels. Nonlinear interaction in the System provides domination of one feedback circuit during some time, but it can cause moving this dominant to the other part of the system where the behavior of the system so different from the first case, that these two situations seem to have nothing in common. Nonlinear functional dependences make Complex Systems extremely sensitive to the majority of its parameters. At the same time, such nonlinear behavior provides the System with resistance to the efforts having the purpose to change its behavior. Together with this, such nonlinearity, being correctly described, allows to model systems with real dynamic characteristics. Complex Systems have characteristics, which, in general case, are unknown. Their behavior strongly differs from the behavior of Simple Systems whose analysis is habitual to human consciousness. Modern science and mathematical modeling mostly analyze the behavior of simple systems.

So a heart, being a biological system can be interpreted as a Complex System consisting of coupled Simple Systems - myocytes which are self - sustaining electrical generators, generating pulses at about $1 \mathrm{HZ}$ frequency. Considering heart as a whole Van der Pol [2] suggested to describe its electrical activity within the framework of the relaxation solution of the Van der Pol equation whereas Norbert Weiner formulated the mathematical model of the process of conduction of impulses in a network of connected excitable elements, such as myocytes in cardiac muscle [3]. Later Ginzburg and Landau [4] using the Van der Pol equations as elements linked in a chain demonstrated in their behavior some different modes of dynamics: regular oscillations, chaotic dynamics and intermittency dynamics of the order-disorder type.

\section{The hypothesis and its mathematical model}

Among the existing papers on heart dynamics modeling $[5,6,7,8,9,10]$ it was not possible to meet ones using Ginzburg Landau chain dynamics as a source of the heart functions modes. Following the suggested hypothesis, a heart can be interpreted as a multi degrees of freedom dynamic system possessing with such

DOI: 10.9790/0661-190302113121 $\quad$ www.iosrjournals.org $113 \mid$ Page


modes of functioning, as regular beatings corresponding to regular oscillations in the Ginzburg Landau chain dynamics, fibrillation, corresponding to the chaotic dynamics of the Ginzburg Landau chain dynamics and ischemia, corresponding to the intermittency of the order - disorder type dynamics of the Ginzburg Landau chain.

Most of cardio pathologies can be described within the framework of the above-mentioned approach through the Fourier spectra feature analysis of the electrocardiograph (ECG) data.

To follow the put forward hypothesis a simple mathematical model has been elaborated. First, it had to describe a regular electrocardiograph activity of a healthy heart ECGs which could be expanded into regular Fourier series. The model included two coupled Van der Pol equations with a time lag [11]. First equation reflected the mentioned Van der Pol's idea [2] about describing electrical pulses of the whole heart through the relaxation solution of the Van der Pol equation. The second equation in the couple described the dynamics of electrical potential of a group of myocytes on the surface of myocardium realizing the Norbert Weiner model of conduction of impulses in a network of myocytes in myocardium [3]. Since the oscillatory processes in a heart have the auto wave character the time lag was introduced into the model in accordance with the theorem proving the possibility of substitution the wave links by the delaying ones [12]. The mathematical model of the first stage of the hypothesis looks in the following way:

$$
\begin{aligned}
& \frac{d^{2} M_{1}}{d t^{2}}-a_{1}\left(1-Y_{1}\right) \frac{d M_{1}}{d t}+\omega_{1}^{2}\left(1+\alpha_{1} M_{2}\right) M_{1}=c_{1} \frac{d^{2} M_{2}}{d t^{2}}+d_{1} F_{1}+d_{2} F_{2} \\
& b_{1} Y_{1}+T_{1} \frac{d Y_{1}}{d t}=M_{1}{ }^{2} \\
& \frac{d^{2} M_{2}}{d t^{2}}-a_{2}\left(1-Y_{2}\right) \frac{d M_{2}}{d t}+\omega_{2}{ }^{2}\left(1+\alpha_{2} M_{1}\right) M_{2}=c_{2} \frac{d^{2} M_{1}}{d t^{2}}+d_{1} F_{1}+d_{2} F_{2} \\
& b_{2} Y_{2}+T_{2} \frac{d Y_{2}}{d t}=M_{2}{ }^{2}
\end{aligned}
$$

Where $M_{1}$ - is the magnitude proportional to the dynamic electric potential of the whole myocardium, $M_{2}$ - is the magnitude proportional to the dynamic electric potential of the myocyte locality on the surface of the myocardium, $Y_{1}$ - is the magnitude proportional to the time lag which requires the propagation of the electric impulse in the myocardium, $Y_{2}$ - is the magnitude proportional to the time lag which requires the propagation of the electric impulse in the myocyte locality, $b_{1}$ - is the magnitude proportional to the square of the myocardium, $b_{2}$ - is the magnitude proportional to the square of the myocyte locality, $T_{1}$ - is the magnitude proportional to the time of the myocardium contraction, $T_{2}$ - is the magnitude proportional to the period of oscillations in the myocyte locality, $\omega_{1}, \omega_{2}$ - are the beating frequencies equal to $1, F_{1}$ - is the perturbation function of the resonant external medium effect on the heart at the frequency of about $1 \mathrm{~Hz}, F_{2}-$ is the perturbation function of the resonant external medium effect on the heart at the frequency of about $20 \mathrm{~Hz}$., $c_{1}, c_{2}, d_{1}, d_{2}, \alpha_{1}, \alpha_{2}$ - are the constants. The computer study of the system (1) shows that in case of intact myocardium and under the absence of external influence at $1 \mathrm{~Hz}$ frequency, the model reproduces the oscillations similar to a regular ECG (Fig. 1) with its Fourier spectrum given in Fig.2.

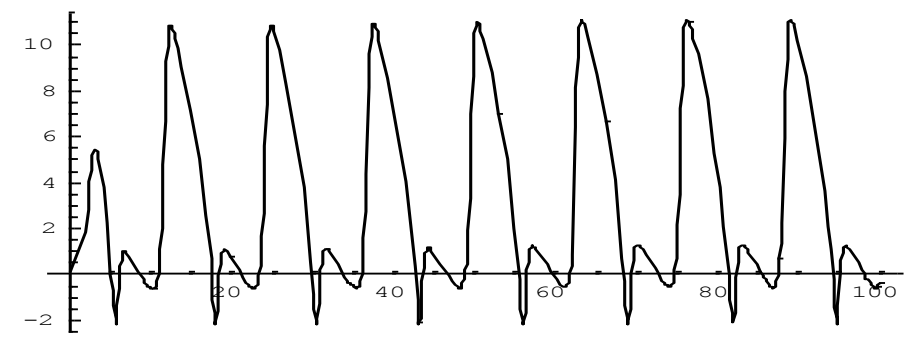

Fig.1. Model of normal ECG generated by the system (1) under the absence of external influence at $1 \mathrm{~Hz}$ frequency. Horiz. Axis - time, Vert.axis - voltage/ Units conditional. 


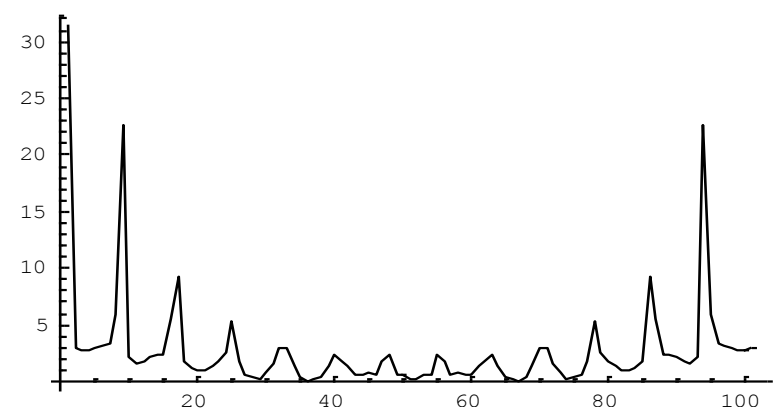

Fig.2. Fourier spectrum of the model ECG shown in Fig.1. Horiz. Axis - frequency, Vert.axis - amplitude. Units conditional.

The obtained data result in a prediction of consequences of resonant external influence at $1 \mathrm{~Hz}$ frequency following from the hypothesis. In particular, an enough intense external resonant influence can discompose the regular model oscillations. The computer study shows that increasing the amplitude of the external perturbation by $50 \%$ brings the breakage of the rhythm of model oscillations corresponding to the state of the heart fibrillation (Fig.3.) This can be called a model infarct. At the same time, the hypothesis predicted the threshold of the external perturbation amplitude that would stop the discomposing of the model.

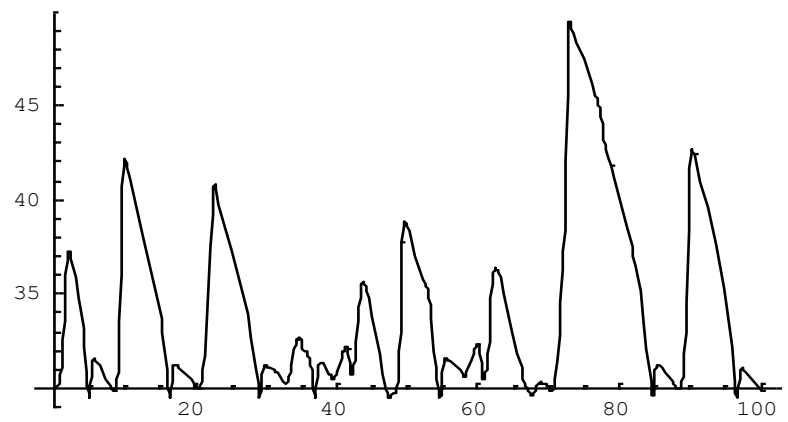

Fig.3. Dynamics of the model ECGs corresponding to the state of the heart fibrillation under the increase of the amplitude of external perturbation. Horiz. Axis - time, Vert.axis - voltage. Units conditional.

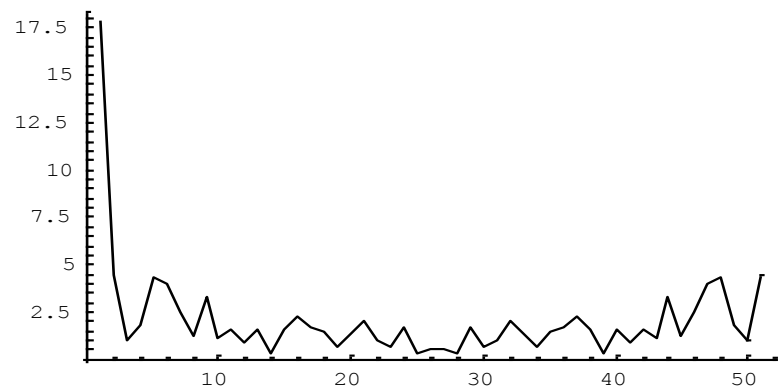

Fig.4. Fourier spectrum of the model fibrillation ECGs (Fig.3.) under the increase of the amplitude of external perturbation. Horiz. Axis - frequency, Vert.axis - amplitude. Units conditional.

The further increase of the external perturbation amplitude resulted in total synchronization of oscillations, reminding the dynamics of heart stimulated by a cardio stimulator (Fig.5).

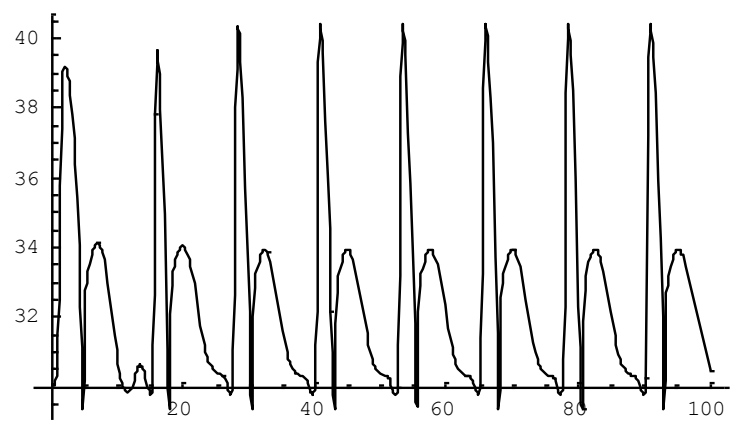

Fig.5. Model ECG in the model mode of "cardio stimulator". . Horiz. Axis - time, Vert.axis - voltage. Units conditional. 


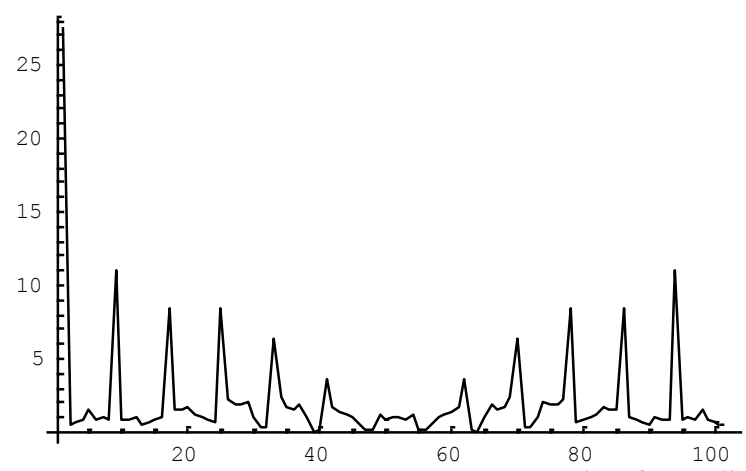

Fig.6. Fourier spectrum of the model ECG (Fig.5.) in the model mode of "cardio stimulator". Horiz. Axis frequency, Vert.axis - amplitude. Units conditional.

Further sophistication of the hypothesis and its model included enlarging the number of coupled equations to link some cardio pathologies with the dynamics of the model therefore forming the next stage of the Ginzburg Landau chain having four coupled Van der Pol equations. The first stage of the mathematical model ( 2 equations) as it was shown above followed the predictions of the hypothesis concerning chaotic states of the model corresponding to the state of heart fibrillation. The second stage (4 equations) brought the intermittency of the order disorder type of the Ginzburg Landau chain dynamics corresponding to the pathological state of heart dynamics - ischemia. The two additional equations of the model described the oscillatory mechanism of the ischemia process and a predictably existing a self-defending mechanism from ischemia in the heart dynamics. The enlarged system of equations of the hypothesis model looked in the following way:

$$
\begin{aligned}
& \frac{d^{2} M_{1}}{d t^{2}}-a_{1}\left(1-Y_{1}\right) \frac{d M_{1}}{d t}+\omega_{1}^{2}\left(1+\alpha_{1} M_{2}+\alpha_{2} M_{3}+\alpha_{3} M_{4}\right) M_{1}=c_{1} \frac{d^{2} M_{2}}{d t^{2}}+c_{2} \frac{d^{2} M_{3}}{d t^{2}}+c_{3} \frac{d^{2} M_{4}}{d t^{2}}+ \\
& d_{1} F_{1}+d_{2} F_{2}-d_{3} F_{3} ; \\
& b_{1} Y_{1}+T_{1} \frac{d Y_{1}}{d t}=M_{1}^{2} \\
& \frac{d^{2} M_{2}}{d t^{2}}-a_{2}\left(1-Y_{2}\right) \frac{d M_{2}}{d t}+\omega_{2}^{2}\left(1+\alpha_{4} M_{1}+\alpha_{5} M_{3}+\alpha_{6} M_{4}\right) M_{2}=c_{4} \frac{d^{2} M_{1}}{d t^{2}}+c_{5} \frac{d^{2} M_{3}}{d t^{2}}+c_{6} \frac{d^{2} M_{4}}{d t^{2}}+ \\
& d_{1} F_{1}+d_{2} F_{2}-d_{3} F_{3} ; \\
& b_{2} Y_{2}+T_{2} \frac{d Y_{2}}{d t}=M_{2}^{2} \\
& \frac{d^{2} M_{3}}{d t^{2}}-a_{3}\left(1-Y_{3}\right) \frac{d M_{3}}{d t}+\omega_{3}^{2}\left(1+\alpha_{7} M_{1}+\alpha_{8} M_{2}+\alpha_{9} M_{4}\right) M_{3}=c_{7} \frac{d^{2} M_{1}}{d t^{2}}+c_{8} \frac{d^{2} M_{2}}{d t^{2}}+c_{9} \frac{d^{2} M_{4}}{d t^{2}}+ \\
& d_{1} F_{1}+d_{2} F_{2}-d_{3} F_{3} ; \\
& b_{3} Y_{3}+T_{3} \frac{d Y_{3}}{d t}=M_{3}^{2} \\
& (2)
\end{aligned}
$$

$$
\begin{aligned}
& \frac{d^{2} M_{4}}{d t^{2}}-a_{4}\left(1-Y_{4}\right) \frac{d M_{4}}{d t}+\omega_{4}{ }^{2}\left(1+\alpha_{10} M_{3}+\alpha_{11} M_{2}+\alpha_{12} M_{1}\right) M_{4}=c_{10} \frac{d^{2} M_{1}}{d t^{2}}+c_{11} \frac{d^{2} M_{2}}{d t^{2}}+c_{12} \frac{d^{2} M_{3}}{d t^{2}}+ \\
& d_{1} F_{1}+d_{2} F_{2}-d_{3} F_{3} ; \\
& b_{4} Y_{4}+T_{4} \frac{d Y_{4}}{d t}=M_{4}{ }^{2}
\end{aligned}
$$

Где $M_{3}$ - is the magnitude proportional to the dynamic electric potential of the cluster defending myocardium from ischemia, $M_{4}$ - is the magnitude proportional to the dynamic electric potential of the ischemia cluster, 
$Y_{3}$ - is the magnitude proportional to the time lag which requires the propagation of the electric impulse in the defending cluster, $Y_{4}$ - is the magnitude proportional to the time lag which requires the propagation of the electric impulse in the ischemia cluster, $b_{3}$ - is the magnitude proportional to the square of the defending cluster, $b_{4}$ - is the magnitude proportional to the square of the ischemia cluster, $T_{3}$ - is the magnitude proportional to the period of group oscillations in the defending cluster, $T_{4}$ - the magnitude proportional to the period of group oscillations in the ischemia cluster, $\omega_{3}$ - is the average frequency in the defending cluster lying in the band of 6-12 Hz, $\omega_{4}$ - the average frequency in the ischemia cluster lying in the band of 14-20 Hz, $F_{3}$ random function reflecting the effect of external negative influence: alcohol, smoking and so on.

Computer study of the enraged model containing in its structure the sub models of the ischemia cluster and the defending cluster demonstrated the high compensating ability of the latter. In particular, under the model decreasing of the myocardium surface by $50 \%$ the state of model fibrillation didn't come, different from the reported above dynamics of the system (1) in the first stage of the model (Fig.3,4), but resulted only in upsetting the model ECG rhythm of the system (2) dynamics in the second stage of the model ( Fig.7,8).

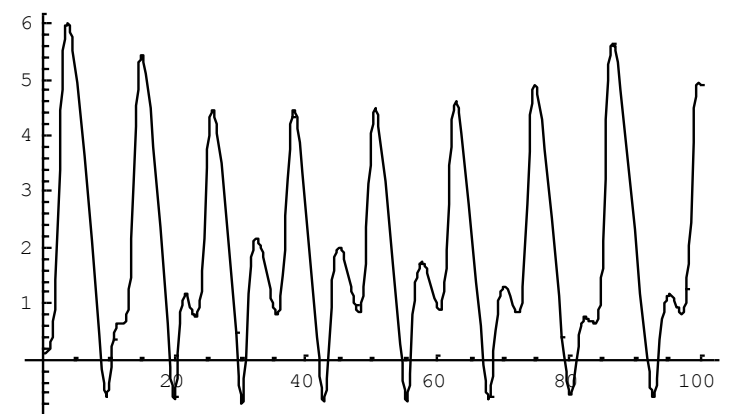

Fig.7. Decomposition of the model ECG rhythm under the model decreasing of the myocardium surface by $50 \%$... Horiz. Axis - time, Vert.axis - voltage. Units conditional.

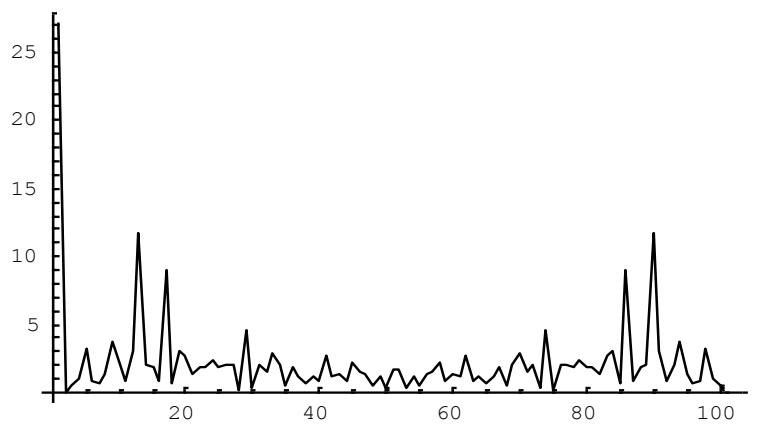

Fig.8. Fourier spectrum of the model ECG under the model decreasing of the myocardium surface by $50 \%$.

Horiz. Axis - frequency, Vert.axis - amplitude. Units conditional.

The computer study of the model (2) in case of existing a developed ischemia demonstrates the process of interaction between the two oscillatory regions- clusters: self-defending and ischemia ones resulting in decreasing the dimensions of the ischemia cluster part in the Fourier spectrum of the model ECG (Fig.9,10).

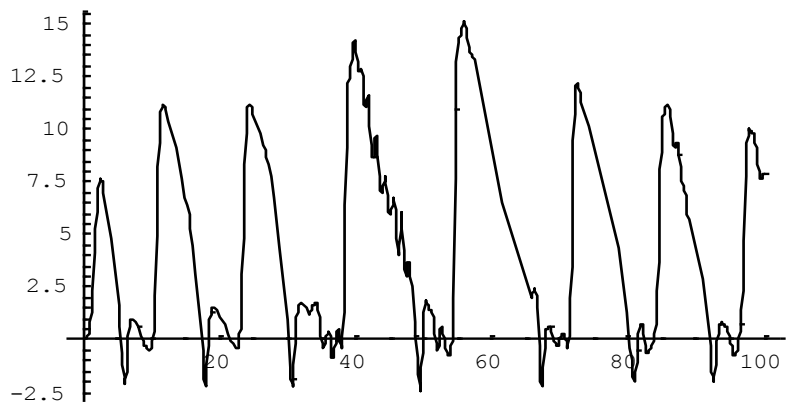

Fig.9. Model ECG reflecting the process of interaction between the ischemia and defending clusters. . Horiz. Axis - time, Vert.axis - voltage. Units conditional. 


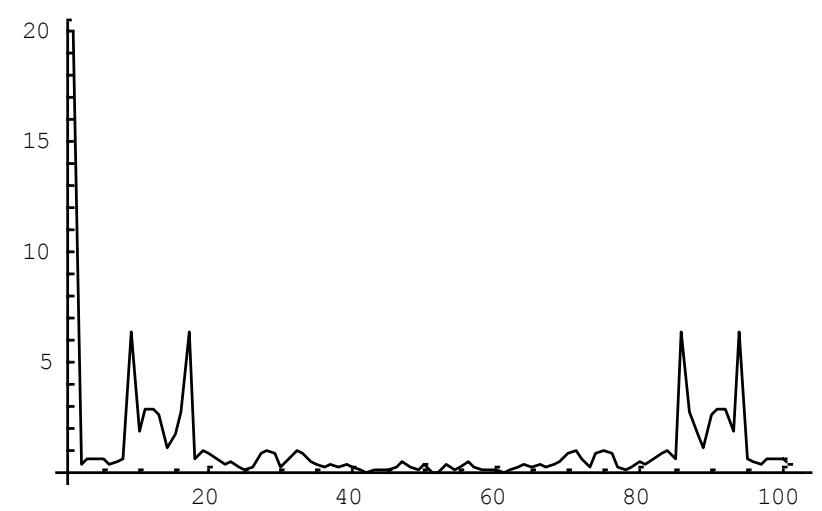

Fig.10. Fourier spectrum of the model ECG in the process of interaction between the ischemia and defending clusters. Horiz. Axis - frequency, Vert.axis - amplitude. Units conditional.

\section{The pilot data supporting the proposed hypothesis}

To verify the proposed hypothesis an electrocardiograph research has been carried out. The study involved 66 people aged 42-60 years with angina (heart failure) of various functional class (FC), including 36 people with typical pain and episodes of SMI (men - 25, women - 11) and 30 (men - 18, women - 12) with the typical pain, without SMI episodes.

The following patients were excluded from the study: a permanent form of atrial fibrillation; complete atrioventricular (a-c) The blockade, complete blockade of the left bundle branch block, the technical causes of false-negative or false-positive detection in the identification of myocardial ischemia (recommendations Holter), life-threatening arrhythmias, acute ischemic stroke, tumors, severe anemia, thyroid disease in the stage of decompensation, lack of informed consent, the inability of the stress tests, the results of stress tests questionable and XM ECG. The diagnosis was based on clinical, medical history, imaging studies. Verification of the diagnosis was carried out using samples with physical exercise (bicycle ergometry (BEM) or stress echocardiography (stress echocardiography)). All patients underwent ECG HM. For the VEM and stress echocardiography was used ergometer Siemens-Elma. Stress echocardiography was performed using a software module Stress-12, the Cardio-automated system for ECG recording in 12 conventional leads (manufacturer ZAO "Diamant", St. Petersburg). Interpretation of changes in ST-segment based on common criteria for ischemic response to the load. Used load threshold power protocol with stepwise increase of its 25 watts every 3 minutes. until the ECG and / or echocardiogram-load criteria for termination or submaximal heart rate (HR). The ECG were registered at healthy patients as well as at patients with differently developed ischemia. The patient with a cardio stimulator has been also studied. In accordance with the first stage mathematical model (1) the mode of real normal heart beatings ECG and its Fourier spectrum (Fig.11) corresponded to the model ECG and its Fourier spectrum shown in the Fig.1,2. The Fourier spectrum of the ECG of heart beatings synchronized by the cardio simulator (Fig.12) corresponded to the model ECG Fourier spectrum generated by the model.

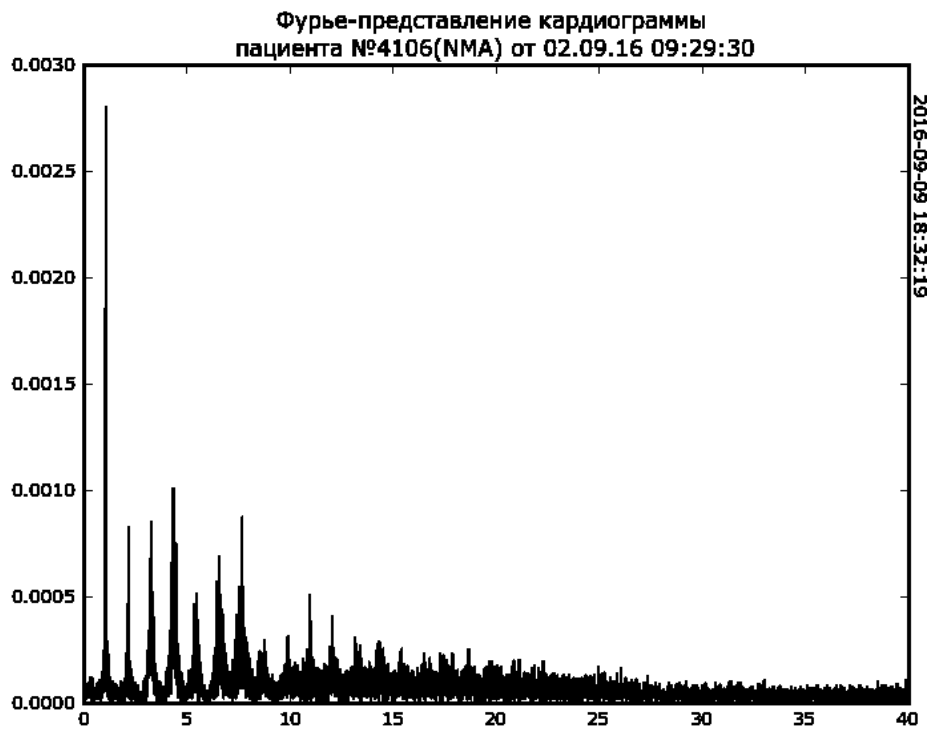

Fig.11. Normal real heart beatings ECG's Fourier spectrum (Fig.11), corresponding to the generated by the model (Fig.2). Horiz. Axis - frequency Hz, Vert.axis -Volt. 


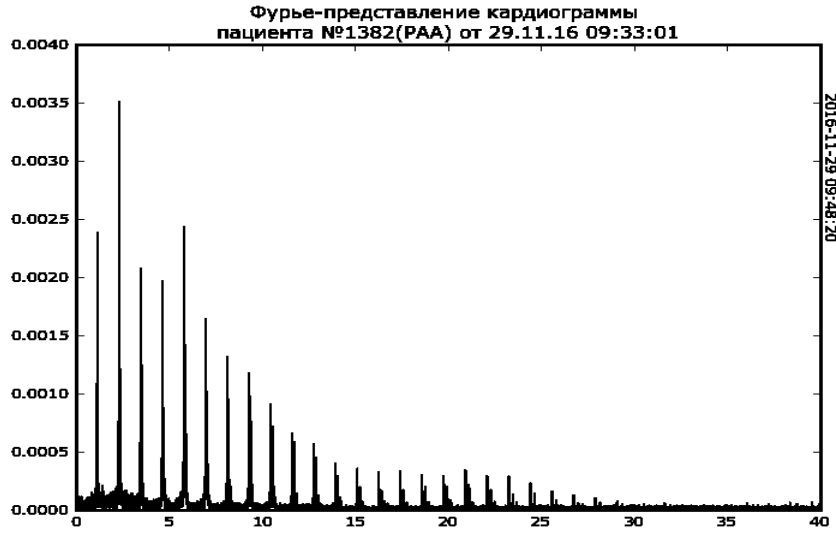

Fig.12. Fourier spectrum of the ECG of the patient heart beatings synchronized by the cardio simulator. Horiz. Axis - frequency $\mathrm{Hz}$, Vert.axis -Volt.

Developed ischemia process in accordance with the hypothesis model represents a forming of an autonomous dynamic system in myocardium, possessing the oscillatory parameters different from those of normal myocardium tissue. In particular, the main frequencies of normal heart beatings lie in the band of 1-12 Hz. whereas the resonant curve of the ischemic heart ECG Fourier spectrum lies in the band of 14-20 Hz. The Fourier spectrum of a patient with a developed ischemic process is shown in Fig.13. Together with this, it was demonstrated during the study of some additional EEG spectra of the same patient that the rate of development of his ischemic process could considerably vary, as it can be seen comparing spectra in Fig.13 and Fig.14. As it can be observed from the ECG spectrum details in Fig.14 the amplitudes of the harmonics in the band of 5-12 $\mathrm{Hz}$ have increased almost twice and the resonance ischemic curve has lost its initial symmetric form (Fig.13) and shifted to the left. In other words, the ischemic process in the spectrum given in the Fig.14 is less expressed than in the spectrum given in the Fig.13. It proves that according to the hypothesis in the dynamics of heart activity there exists a defending mechanism restricting the development of ischemic process through lowering its oscillatory energy. This role can play only an additional oscillatory system existing in the heart dynamics.

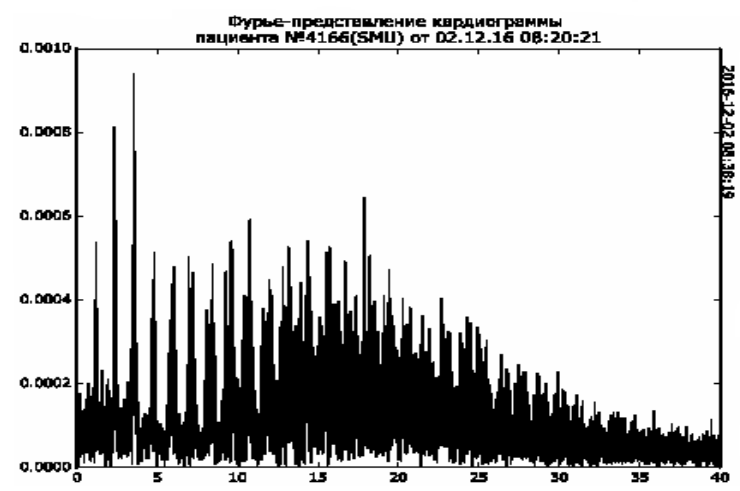

Fig.13. Fourier spectrum of a patient's EEG with a developed ischemic process. Horiz. Axis - frequency Hz, Vert.axis -Volt..

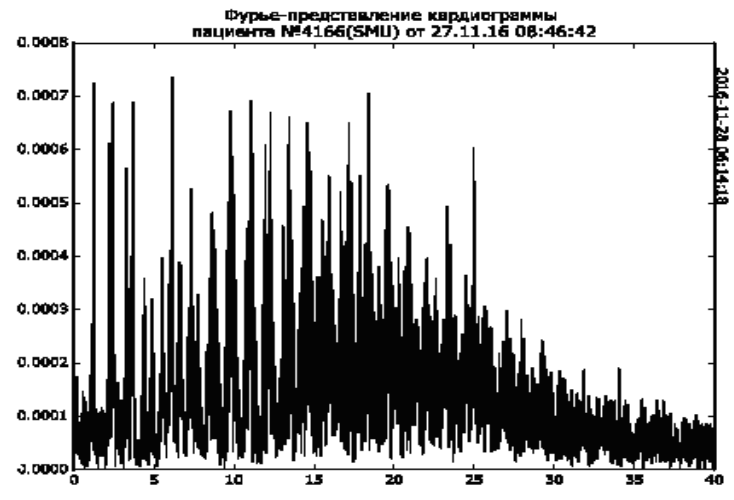

Fig.14. ECG Fourier spectrum of the patient with developed ischemia during spontaneous decrease of the volume of his ischemic process. Horiz. Axis - frequency Hz, Vert.axis -Volt. 
The study of the ECG Fourier spectra of a patient with not developed ischemic process manifested the same general picture as in Fig.13 with a resonant curve but with o lower level of oscillatory energy in its spectrum to compare with Fig.13.

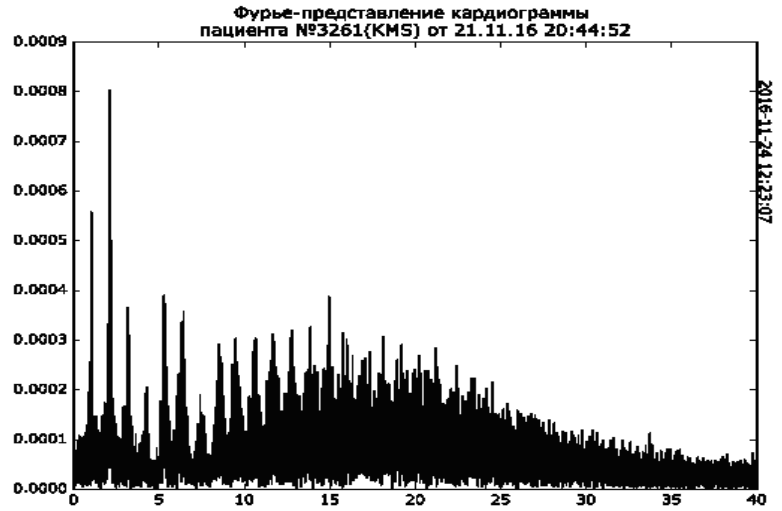

Fig.15.ECG Fourier spectra of a patient with not developed ischemic process. Volt.

Consider the dynamics of not developed ischemic process. As in the case of developed ischemic process (Fig.13) the dimension of the ischemic locality could also vary spontaneously as it can be seen, comparing the Fig.15 and Fig.16. Horiz. Axis - frequency Hz, Vert.axis - Volt.

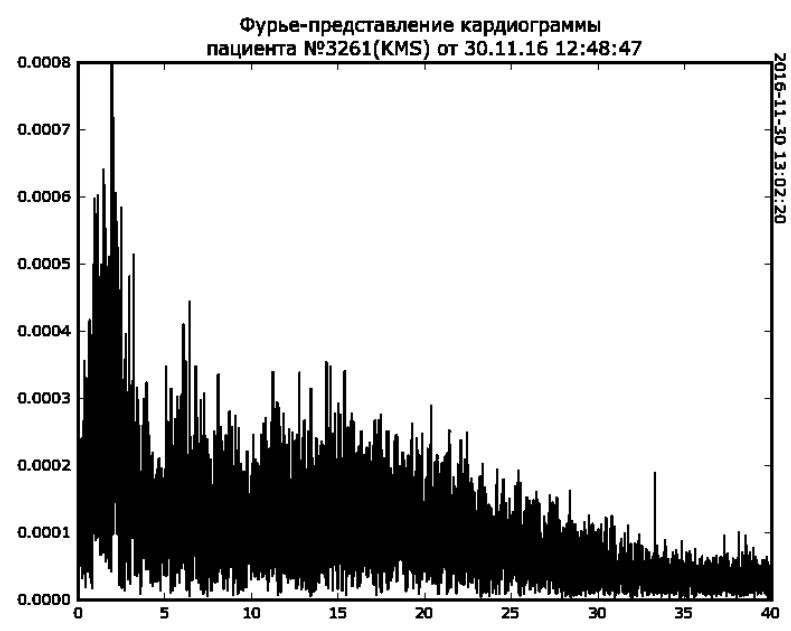

Fig.16. ECG Fourier spectrum of the patient with not developed ischemia during spontaneous decrease of the volume of his ischemic process. Horiz. Axis - frequency Hz, Vert.axis - Volt.

As it can be concluded from the details of the ECG spectrum shown in Fig.16 the amplitudes of the ischemic process harmonics in the band of $14-20 \mathrm{~Hz}$ remained almost unchanged whereas the resonance curve of the process has lost its symmetry and shifted sharply to the left. In other words, the ischemic process in the spectrum given in the Fig.16 is less expressed than in the spectrum given in the Fig.15.

\section{Conclusion and discussion}

The suggested hypothesis that a heart can be interpreted as a multi degrees of freedom Complex System, possessing with such modes of functioning as regular beatings corresponding to regular oscillations in the Ginzburg Landau chain dynamics, fibrillation corresponding to the chaotic dynamics of the Ginzburg Landau chain and ischemia corresponding to the intermittency of the order - disorder type dynamics of the Ginzburg Landau chain has been confirmed through developing and computer study of the mathematical model consisting of four coupled Van der Pol equations with a time lag. The proposed concept brings the necessity to record the ECGs of patients periodically evaluating their current cardio logical state and assessing the effect of the given therapy. Besides, the above mentioned EEG measuring policy allows to predict an aggravation of the disease and correct its flowing. The model prediction of external perturbation effect on heart dynamics suggests a possibility of using acoustic or electromagnetic generators as therapeutic devices in cardiology. 


\section{References}

[1] Forrester. J. World Dynamics. Wright Allen Press. ISBN 5-17- 019253-3. 1971, p- 384

[2] Van der Pol B, Van der Mark M. Le battement du coeur considere comme oscillation de relaxation et un model elecrtrique du coeur. L'Onde electrique, $1928, \mathrm{~N} 7$.

[3] Wiener $\mathrm{N}$. The mathematical formulation of the problem of conduction of impulses in a network of connected excitable elements, specifically in cardiac muscle / N.

[4] Wiener, A. Rosenblueth // Arch. Del. Instit. De Cardiologia De Mexico, 1946.P. . 205 - 265.

[5] Gaponov - Grehov A.V. and Rabinowitz M.I. Autostructures, Chaotic

[6] Dynamics of Ensembles. In Non linear waves. Ed. "Nauka", Moscow, 1987,p-347. In Russian

[7] Luo C.H. A Dynamic Model of the Cardiac Ventricular Action Potential. I. Simulations of Ionic Currents and Concentration Changes / C.H. Luo, Y. Rudy // Circ Res. 1994. - Vol. 74, N 6. - P. 1071 -1167.

[8] Holden A. V. Computational Biology of Propagation in Excitable Media Models of Cardiac Tissue / A. V. Holden, V.N. Biktashev // Chaos Solitons and Fractals.-2002.-N 13.-P. 1643-1658.

[9] Simeliusa K. Modeling Cardiac Ventricular Activation / K. Simeliusa, J. Nenonena, M. Horacek // International Journal of Bioelectromagnetism. -2001. -N 2. P. 51 - 58

[10] Aliev R.R. A simple model of cardiac excitation / R.R. Aliev, A.V. Panfilov // Chaos, Solutions \& Fractals. 1996. -№3. -P.293-301.

[11] Moe G. A computer model of atrial fibrillation / G. Moe, W. Reinbold, J. Abildskov // Am Heart J. 1964. - N 67. - P. 200-220.

[12] Andreev S.U., Batalov R.E., Popov S.B. Computer Model of the Impulse Propagation in Myocardium. Proceedings of Russian National Congress of Cardiologists. Tomsk, 2004, p 26.

[13] Philip R and Scott. jr. Symmetrical Oscillator Having Unsymmetrical Solutions. Proceedings of IEEE, volume 64, number 5, May, 1976.

[14] Rubanik V.P. Oscillations of Quasi Linear Systems with a Time Lag. Ed. "Nauka”, Moscow, 1969, p-288. In Russian. 